Proc. XIX International School of Semiconducting Compounds, Jaszowiec 1990

\title{
RECOMBINATION PROCESSES FOR DEEP IMPURITY STATES DEGENERATE WITH THE CONDUCTION BAND*
}

\author{
K. Świątek, M. Godlewski, Z. Kaliński and H. Przybylińska \\ Institute of Physics, Polish Academy of Sciences, Al. Lotników 32/46, Warszawa, Poland
}

(Received August 8, 1990)

We report on the studies of autoionization efficiency and the relevant recombination mechanism for $\mathrm{Eu}^{2+}$ in $\mathrm{Ca}_{x} \mathrm{Cd}_{1-x} \mathrm{~F}_{2}$ depending on the energy level position of $\mathrm{Eu}^{2+}$ excited states in respect to the conduction band states.

PACS numbers: $78.50 .-\mathrm{w}, 76.30 . \mathrm{Kg}, 78.55 . \mathrm{Hx}$

Lattice relaxation phenomena are of current technological and scientific interest as evidenced by the large number of review papers published recently [1-4]. They lead to such effects as metastability or bistability even in covalent crystals, e.g. EL2 defect in GaAs, DX centers in GaAlAs [4], thermal donors in silicon [3] etc. In this paper we study the influence of lattice relaxation on the autoionization and recombination processes from the excited states of $\mathrm{Eu}^{2+}$ in $\mathrm{Ca}_{x} \mathrm{Cd}_{1-x} \mathrm{~F}_{2}$. The $\mathrm{Eu}^{2+}\left(4 f^{7}\right)$ ion forms a relatively shallow donor with $0.33 \mathrm{eV}$ thermal ionization energy in $\mathrm{CdF}_{2}$ and a very deep center (with the energy level located more than $4 \mathrm{eV}$ below the conduction band) in $\mathrm{CaF}_{2}$. Depending on the composition $x$ the $4 f^{6} 5 d^{1}$ (e) excited states of $\mathrm{Eu}^{2+}$, extending from $3 \mathrm{eV}$ to $3.7 \mathrm{eV}$ above the ground state, are totally or partly degenerate with the continuum of conduction band (CB) states, or are located within the forbidden gap. The emphasis of this paper is put on the correlation between the recombination mechanism and the position of the excited states in respect to the CB states.

A unique property of $\mathrm{CdF}_{2}$ is the extremely large lattice relaxation concomitant with the change of the charge state of a deep center [5]. For $\mathrm{Eu}^{2+}$ the lattice relaxation energy is estimated to be about $2.4 \mathrm{eV}$. In consequence, the $4 f^{7} \rightarrow 4 f^{6} 5 d^{1}$ intra-ion absorption is superimposed on a very weak photoionization background and well resolved (Fig. 1). The resonant nature of the excited states was proved by a unique photo-EPR experiment discussed in a previous paper [6]. The appropriate configuration coordinate diagram is shown in Fig. 2a.

*This work was supported by CPBP 01.12. 


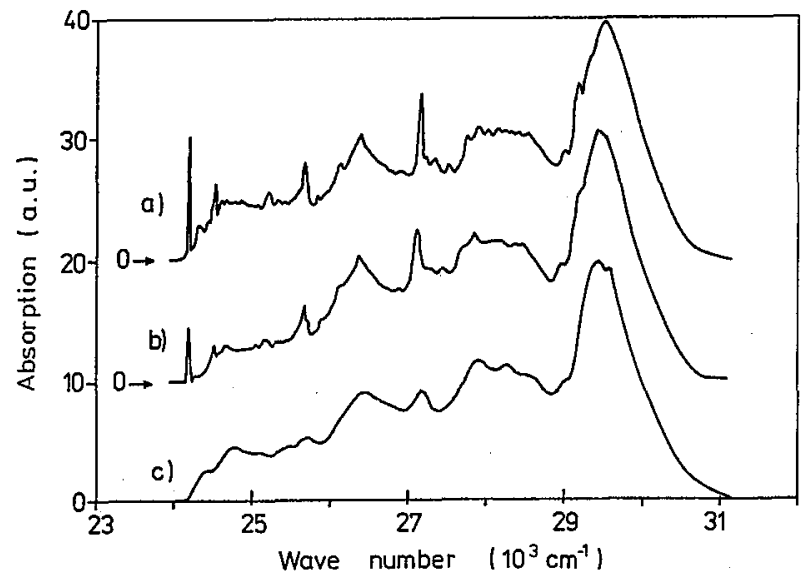

Fig. 1. Absorption spectra of the $4 f^{7} \rightarrow 4 f^{6}\left({ }^{7} F_{J}\right) 5 d^{1}(\mathrm{e})$ transition of $\mathrm{Eu}^{2+}(\mathrm{J}=$ $0,1, \ldots, 6)$ in $\mathrm{Ca}_{x} \mathrm{Cd}_{1-x} \mathrm{~F}_{2}$ mixed crystals, measured at $4.2 \mathrm{~K}: \mathrm{a}$ ) for $x=1, \mathrm{~b}$ ) for $x=0.7$, c) for $x=0$.

The measurements were performed on $\mathrm{Ca}_{x} \mathrm{Cd}_{1-x} \mathrm{~F}_{2}$ crystals grown by the Bridgman-Stockbarger method with $\mathrm{EuF}_{3}$ added to the melt. The conversion to the $2+$ charge state of $\mathrm{Eu}$ was achieved by annealing in $\mathrm{H}_{2}$ vapor. The absorption spectra due to the $4 f^{7} \rightarrow 4 f^{6} 5 d^{1}$ (e) transitions of $\mathrm{Eu}^{2+}$ in $\mathrm{Ca}_{x} \mathrm{Cd}_{1-x} \mathrm{~F}_{2}$ for various compositions $x$ are shown in Fig. 1. The characteristic feature of these spectra is the Fano broadening [7] of the lines corresponding to transitions to those excited states which are degenerate with the continuum of CB states. The composition at which the lowest excited state enters the continuum can be precisely determined as $x=0.65$. For $x=0.7$ the lowest state is already located below the CB minimum and, as can be seen in Fig. 1, the zero-phonon line is unbroadened as compared to $\mathrm{CaF}_{2}$. From the Fano broadening the autoionization lifetime can be determined, and it can be seen that it depends on the position of the excited level in respect to the CB. For $x=0$ the average autoionization lifetime $\tau$ is $\sim 60$ fs, whereas for $x=0.7 \tau \approx 500 \mathrm{fs}$ (moreover, the broadening is larger the higher the level). It is evident that for higher compositions there are energy barriers for autoionization due to lattice relaxation and the barrier height decreases with increasing energy of the level in respect to the CB minimum.

In $\mathrm{CaF}_{2}$ the intra-ion excitation of $\mathrm{Eu}^{2+}$ is followed by a characteristic blue emission (Fig. 2b). The question is what is the recombination mechanism when the excited states of $\mathrm{Eu}^{2+}$ are degenerate with the continuum of CB states and how does it affect the luminescence. The emission spectra of $\mathrm{Ca}_{x} \mathrm{Cd}_{1-x} \mathrm{~F}_{2}: \mathrm{Eu}^{2+}$ crystals under excitation to the highest excited state of the $4 f^{6} 5 d^{1}(q)$ configuration for various compositions $x$ are shown in Fig. 2b. It can be seen that as long as the lowest excited state is located in the forbidden gap the character of the $\mathrm{Eu}^{2+}$ emission does not change, even if (as for $x=0.7$ ) all the higher lying states are 

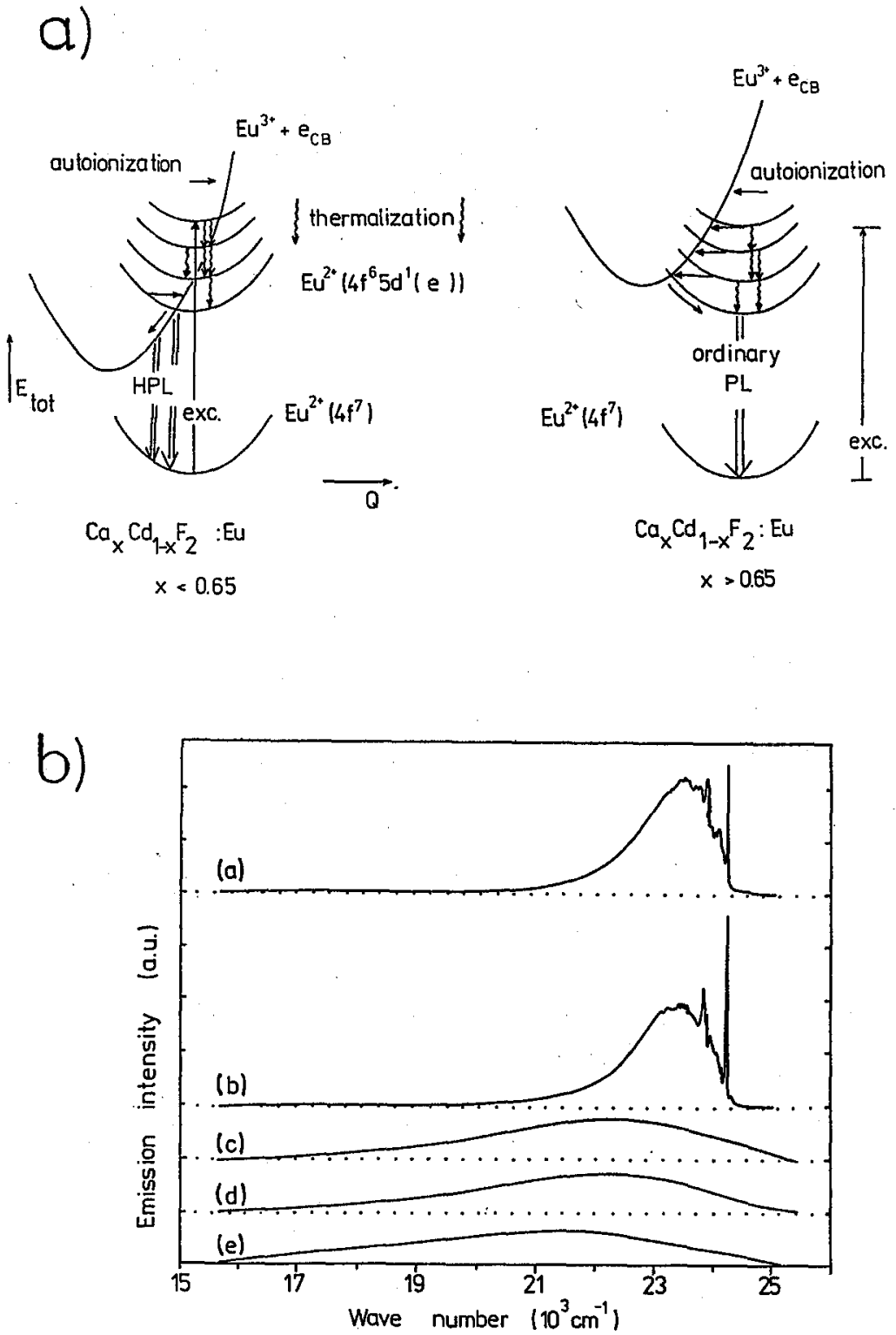

Fig. 2. a) The configuration coordinate diagram of the absorption and recombination transitions for the isolated $\mathrm{Eu}^{2+}$ center in the $\mathrm{Ca}_{x} \mathrm{Cd}_{1-x} \mathrm{~F}_{2}$ lattice for $x<0.65$ and $x>0.65$; b) the photoluminescence spectra of the $\mathrm{Ca}_{x} \mathrm{Cd}_{1-x} \mathrm{~F}_{2}$ : Eu crystals under the $4 f^{7} \rightarrow 4 f^{6}\left({ }^{7} F_{6}\right) 5 d^{1}(\mathrm{e})$ excitation $(340 \mathrm{~nm})$, measured at $4.2 \mathrm{~K}$ for: a) $\left.x=1, \mathrm{~b}\right) x=0.7$, c) $x=0.5$, d) $x=0.3$, and e) $x=0$. 
already resonant. It means that for $0.7 \leq x \leq 1$ the autoionization lifetime is still comparable to the thermalization within the excited multiplet. The character of the luminescence spectrum drastically changes when the lowest excited state enters the CB. The intra-ion emission is no longer observed, instead a new, broad and featureless emission band appears. The high energy threshold of this band coincides with the energy of the zero-phonon line of the $\mathrm{Eu}^{2+}$ intra-ion transition and is composition independent. This coincidence suggests that the broad emission band is also $\mathrm{Eu}^{2+}$-related. The properties of this band are not consistent with the so-called anomalous emission observed for $\mathrm{Eu}^{2+}$ in $\mathrm{BaF}_{2}$ [8] or $\mathrm{Yb}$ in $\mathrm{SrF}_{2}$ [9], i.e. with the bound exciton recombination. We tentatively attribute this new emission to radiative capture of the ionized carrier by $\mathrm{Eu}^{3+}$. Due to very large electron excess energy in the case of low compositions the observed emission includes also hot luminescence (HL). An analogous HL was observed for, e.g. F centers in ionic crystals [10].

\section{References}

[1] G.D. Watkins, Mat. Science Forum 38/41, 39 (1989).

[2] A. Chantre, Appl. Phys. 48, 3 (1989).

[3] J. Hage, P. Wagner, Mat. Science Forum 38/41, 487 (1989).

[4] J.M. Langer, J.E. Dmochowski, L. Dobaczewski, W. Jantsch, G. Brunthaler, Solid State Phenomena, Vol. 10, Sci. Tech. Publ., Paris 1990 , p. 123.

[5] J.M. Langer, Lecture Notes in Physics, Vol. 122, Springer Verlag, Berlin 1980, p. 123.

[6] M. Godlewski, D. Hommel, J.M. Langer, H. Przybylińska, J. Lumin. 24/25, 217 (1981).

[7] U. Fano, J.W. Cooper, Phys. Rev. A 137, 1364 (1965).

[8] C. Pedrini, B. Moine, B. Courtois, D.S. McClure, in Excited States of Transition Metal Elements, ed. B. Jeżowska-Trzebiatowska, J. Legendziewicz, W. Stręk, World Scientific, Singapore 1989, p. 385.

[9] D.S. McClure, C. Pedrini, Phys. Rev. B 32, 8465 (1985).

[10] Y. Mori, R. Hattori, H. Ohkura, J. Phys. Soc. Jpn. 51, 2713 (1982). 